\title{
Analysis of Boundary Layer Flow and Heat Transfer along a Stretching Cylinder in a Porous Medium
}

\author{
Swati Mukhopadhyay \\ Department of Mathematics, The University of Burdwan, Burdwan 713104, India \\ Correspondence should be addressed to Swati Mukhopadhyay, swati_bumath@yahoo.co.in
}

Received 26 March 2012; Accepted 17 April 2012

Academic Editors: G. L. Aranovich and C. D. Daub

Copyright () 2012 Swati Mukhopadhyay. This is an open access article distributed under the Creative Commons Attribution License, which permits unrestricted use, distribution, and reproduction in any medium, provided the original work is properly cited.

\begin{abstract}
This paper presents an axi-symmetric laminar boundary layer flow of a viscous incompressible fluid and heat transfer towards a stretching cylinder embedded in a porous medium. The partial differential equations corresponding to the momentum and heat equations are converted into highly nonlinear ordinary differential equations with the help of similarity transformations. Numerical solutions of these equations are obtained by shooting method. It is found that the velocity decreases with increasing permeability parameter. The skin friction as well as the heat transfer rate at the surface is larger for a cylinder compared to a flat plate.
\end{abstract}

\section{Introduction}

The study of hydrodynamic flow and heat transfer over a stretching cylinders or flat plates has gained considerable attention due to its applications in industries and important bearings on several technological processes. Crane [1] investigated the flow caused by the stretching of a sheet. Many researchers such as Gupta and Gupta [2], Dutta et al. [3], Chen and Char [4] extended the work of Crane [1] by including the effect of heat and mass transfer analysis under different physical situations. Recently, various aspects of such problem have been investigated by many authors such as $\mathrm{Xu}$ and Liao [5], Cortell [6, 7], Hayat et al. [8] and Hayat and Sajid [9].

Flow over cylinders is considered to be two-dimensional if the body radius is large compared to the boundary layer thickness. On the other hand for a thin or slender cylinder, the radius of the cylinder may be of the same order as that of the boundary layer thickness. Therefore, the flow may be considered as axi-symmetric instead of two-dimensional [10-12]. The study of steady flow in a viscous and incompressible fluid outside of a stretching hollow cylinder in an ambient fluid at rest has been done by Wang [13]. The effect of slot suction/injection over a thin cylinder as studied by Datta et al. [10] and Kumari and Nath [12] may be useful in the cooling of nuclear reactors during emergency shutdown, where a part of the surface can be cooled by injecting a coolant (Ishak et al. [14]). Lin and Shih $[15,16]$ considered the laminar boundary layer and heat transfer along horizontally and vertically moving cylinders with constant velocity and found that the similarity solutions could not be obtained due to the curvature effect of the cylinder. Ishak and Nazar [17] showed that the similarity solutions may be obtained by assuming that the cylinder is stretched with linear velocity in the axial direction and claimed that their study may be regarded as the extension of the papers by Grubka and Bobba [18] and Ali [19], from a stretching sheet to a stretching cylinder.

The study of hydrodynamic flow and heat transfer in porous medium becomes much more interesting due to its vast applications on the boundary layer flow control. Heat removal from nuclear fuel debris, underground disposal of radioactive waste material, storage of food stuffs and exothermic and/or endothermic chemical reactions and dissociating fluids in the packed-bed reactors, and so forth are some 


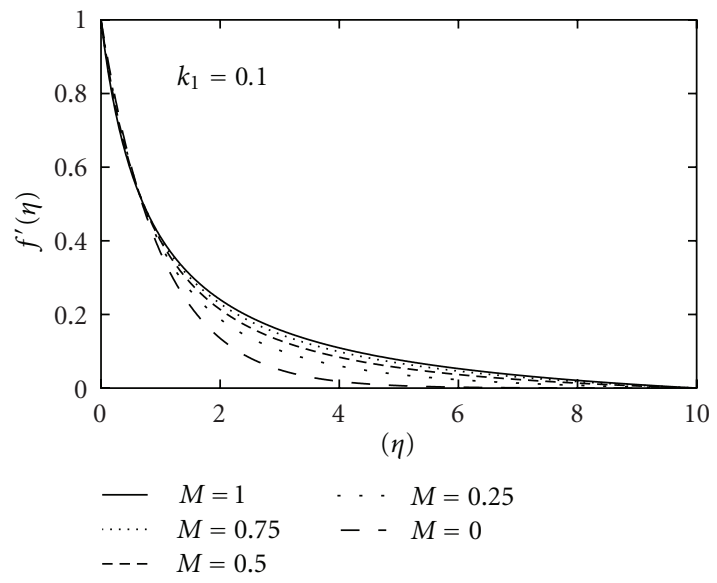

(a)

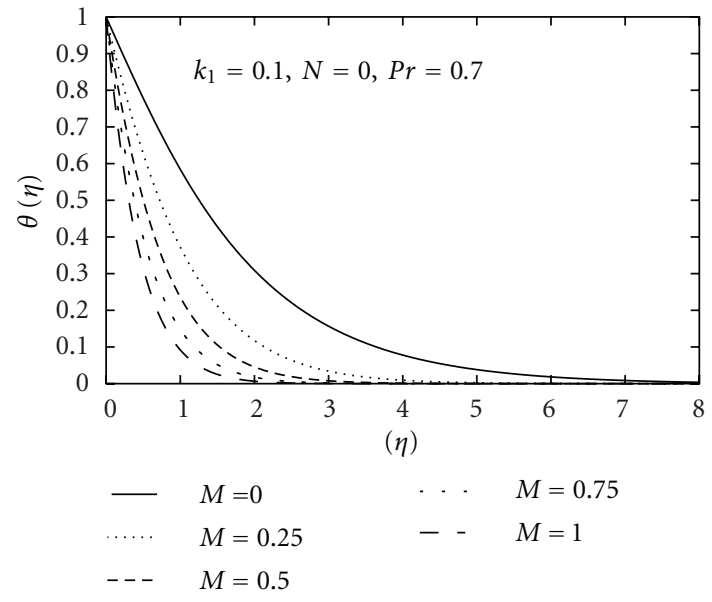

(b)

FIgURE 1: (a) Variation of velocity $f^{\prime}(\eta)$ with $\eta$ for several values of curvature parameter $M$ of the stretching cylinder. (b) Variation of temperature $\theta(\eta)$ with $\eta$ for several values of curvature parameter $M$ of the stretching cylinder.

porous media applications. It is well known that Darcy's law is an empirical formula relating the pressure gradient, the bulk viscous fluid resistance and the gravitational force for a forced convective flow in a porous medium. Deviations from Darcy's law occur when the Reynolds number based on the pore diameter is within the range of 1 to 10 (Ishak et al. [20]).

No attempt has been made yet to analyze the flow and thermal characteristics of boundary layer axi-symmetric flow and heat transfer along a stretching cylinder in a porous medium. Therefore, an attempt is made to study the steady forced convection flow and heat transfer past a stretching cylinder placed in a fluid-saturated porous medium using the Darcy model. Using similarity transformation, a third order ordinary differential equation corresponding to the momentum equation and a second-order ordinary differential equation corresponding to heat equation are derived. Using shooting method, numerical calculations up to desired level of accuracy were carried out for different values of dimensionless parameters of the problem under consideration for the purpose of illustrating the results graphically. The results obtained are then compared with those of Grubka and Bobba [18], Ali [19], and Ishak and Nazar [17] who reported the results for some special case of the presented study. The analysis of the results obtained shows that the flow field is influenced appreciably by the permeability parameter. Estimation of skin friction and heat transfer coefficient which are very important from the industrial application point of view are also presented in the analysis. It is hoped that the results obtained will not only provide useful information for applications but also serve as a complement to the previous studies.

\section{Equations of Motion}

Consider the steady axi-symmetric flow of an incompressible viscous fluid along a stretching cylinder embedded in a porous medium. The continuity, momentum, and energy equations governing such type of flow are written as

$$
\begin{gathered}
\frac{\partial(r u)}{\partial x}+\frac{\partial(r v)}{\partial r}=0, \\
u \frac{\partial u}{\partial x}+v \frac{\partial u}{\partial r}=\frac{v}{r} \frac{\partial}{\partial r}\left(r \frac{\partial u}{\partial r}\right)-\frac{v}{k} u, \\
u \frac{\partial T}{\partial x}+v \frac{\partial T}{\partial r}=\frac{\kappa}{r} \frac{\partial}{\partial r}\left(r \frac{\partial T}{\partial r}\right),
\end{gathered}
$$

where $u$ and $v$ are the components of velocity, respectively, in the $x$ and $r$ directions, $v=\mu / \rho$ is the kinematic viscosity, $\rho$ is the fluid density (assumed constant), $\mu$ is the coefficient of fluid viscosity, $k$ is the porosity of the medium, $\kappa$ is the thermal diffusivity of the fluid, and $T$ is the fluid temperature.

2.1. Boundary Conditions. The appropriate boundary conditions for the problem are given by

$$
\begin{gathered}
u=U(x), \quad v=0, \quad T=T_{w}(x), \quad \text { at } r=R, \\
u \longrightarrow 0, \quad T \longrightarrow T_{\infty}, \quad \text { as } r \longrightarrow \infty .
\end{gathered}
$$

Here, $U(x)=U_{0}(x / L)$ is the stretching velocity, $T_{w}(x)=$ $T_{\infty}+T_{0}(x / L)^{N}$ is the prescribed surface temperature, $U_{0}, T_{0}$ are the reference velocity and temperature, respectively, $T_{\infty}$ is the ambient temperature, $L$ is the characteristic length, and $N$ is the temperature exponent.

2.2. Method of Solution. The continuity equation is automatically satisfied by the introduction of stream function $\psi$ as $u=(1 / r)(\partial \psi / \partial r), v=-(1 / r)(\partial \psi / \partial x)$. Introducing the similarity variables as

$$
\begin{gathered}
\eta=\frac{r^{2}-R^{2}}{2 R}\left(\frac{U}{v x}\right)^{1 / 2}, \quad \psi=(U v x)^{1 / 2} R f(\eta), \\
\theta(\eta)=\frac{T-T_{\infty}}{T_{w}-T_{\infty}}
\end{gathered}
$$




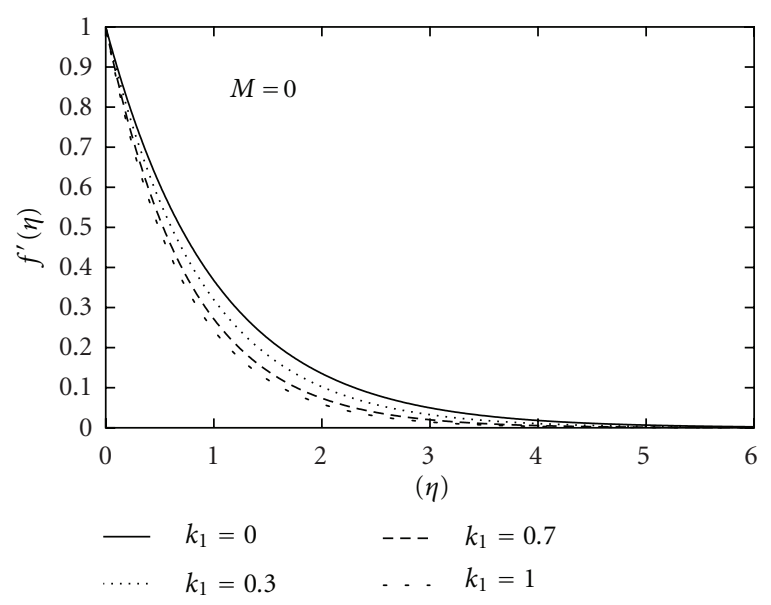

(a)

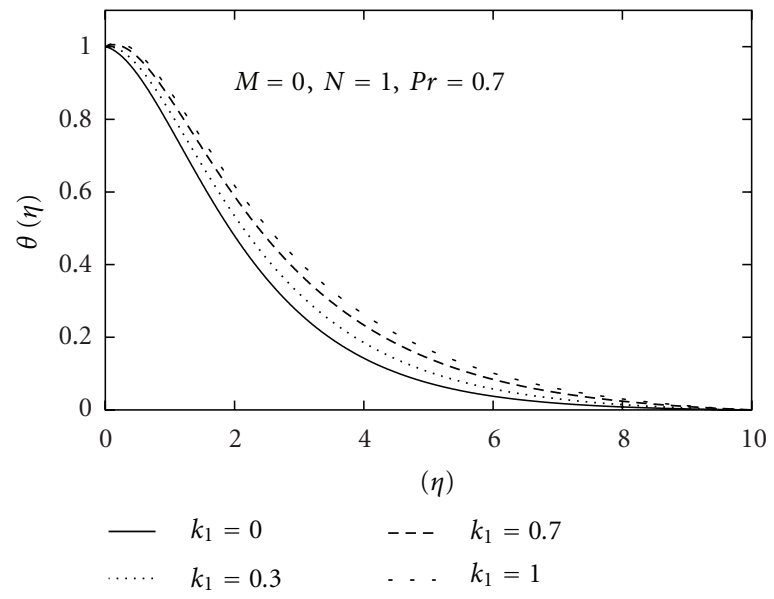

(c)

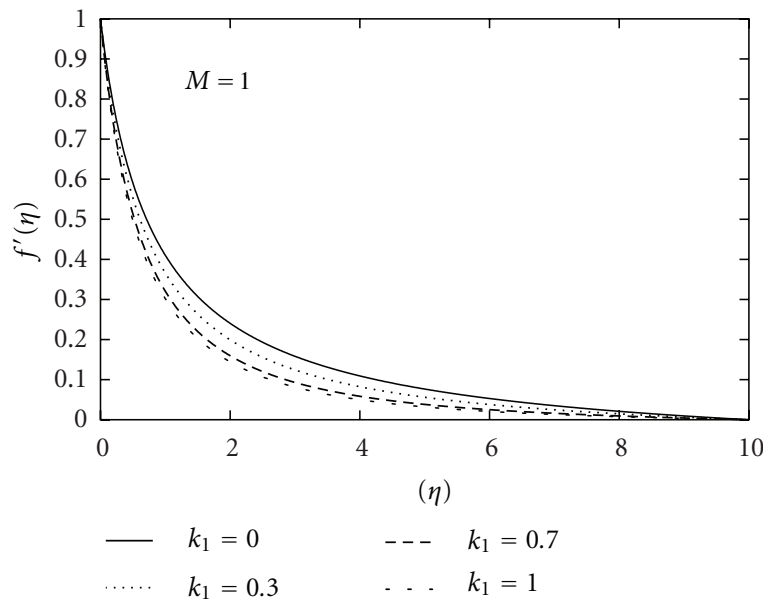

(b)

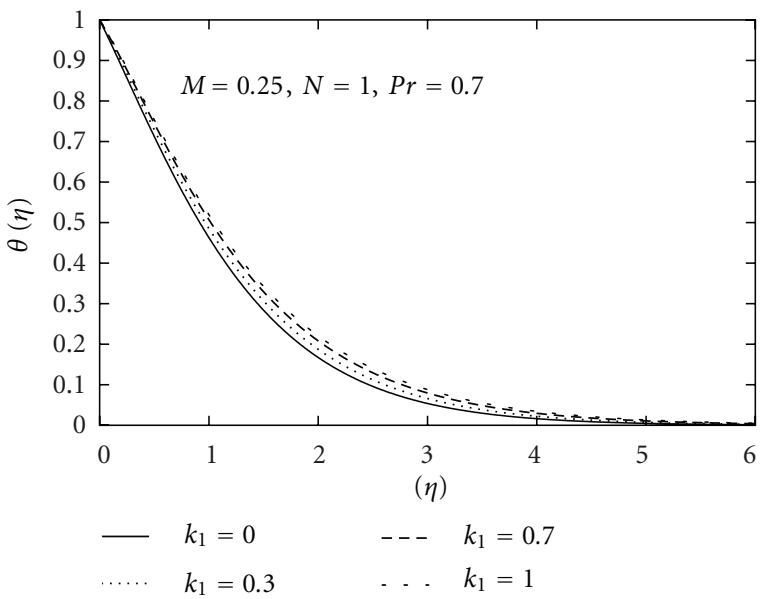

(d)

Figure 2: (a) Variation of velocity $f^{\prime}(\eta)$ with $\eta$ for several values of Permeability parameter $k_{1}$ for flat plate with $M=0$. (b) Variation of velocity $f^{\prime}(\eta)$ with $\eta$ for several values of permeability parameter $k_{1}$ for stretching cylinder with $M=1$. (c) Variation of temperature $\theta(\eta)$ with $\eta$ for several values of permeability parameter $k_{1}$ for flat plate with $M=0$. (d) Variation of temperature $\theta(\eta)$ with $\eta$ for several values of permeability parameter $k_{1}$ for stretching cylinder with $M=0.25$.

and upon substitution of (4) in (2) and (3), the governing equations and the boundary conditions reduce to

$$
\begin{gathered}
(1+2 M \eta) f^{\prime \prime \prime}+2 M f^{\prime \prime}+f f^{\prime \prime}-f^{\prime 2}-k_{1} f^{\prime}=0, \\
(1+2 M \eta) \theta^{\prime \prime}+2 M \theta^{\prime}+\operatorname{Pr}\left(f \theta^{\prime}-N f^{\prime} \theta\right)=0, \\
f^{\prime}=1, \quad f=0, \quad \theta=1 \quad \text { at } \eta=0, \\
f^{\prime} \longrightarrow 0, \quad \theta^{\prime} \longrightarrow 0 \quad \text { as } \eta \longrightarrow \infty,
\end{gathered}
$$

where the prime denotes differentiation with respect to $\eta$, $k_{1}=v L / U_{0} k$ is the permeability parameter of the porous medium and $M=\left(v L / U_{0} R^{2}\right)^{1 / 2}$ is the curvature parameter. The case of nonporous medium is recovered for $k_{1}=0 . k_{1}^{-1}$ will reflect the effect of Darcian flow on the present problem.

One can note that if $M=0$ (i.e., $R \rightarrow \infty$ ), the problem under consideration (with $k_{1}=0$ ) reduces to the boundary layer flow along a stretching flat plate considered by Ali [19], with $m=1$ in that paper. Moreover, when $M=0$ (stretching flat plate) subjected to (6) with $k_{1}=0$ (i.e., for non-porous medium), the analytical solutions of (5) are given by Crane [1] and Grubka and Bobba [18], respectively.

\section{Numerical Method for Solution}

The above equations (5) along with boundary conditions are solved by converting them to an initial value problem. We set

$$
\begin{gathered}
f^{\prime}=z, \quad z^{\prime}=p, \quad p^{\prime}=\frac{\left[z^{2}+k_{1} z-f p-2 M p\right]}{(1+2 M \eta)}, \\
\theta^{\prime}=q, \quad q^{\prime}=-\frac{[\operatorname{Pr}(f q-N z \theta)+2 M q]}{(1+2 M \eta)},
\end{gathered}
$$




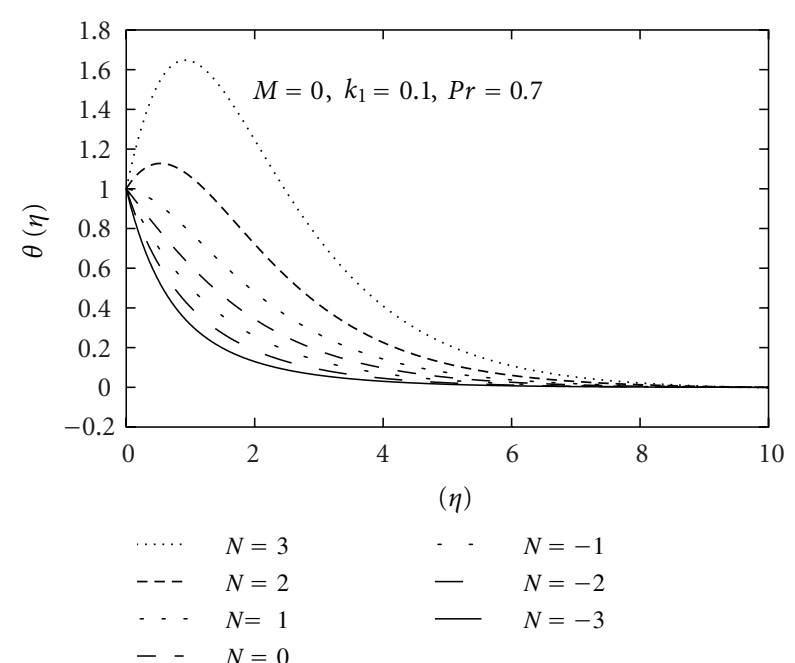

(a)

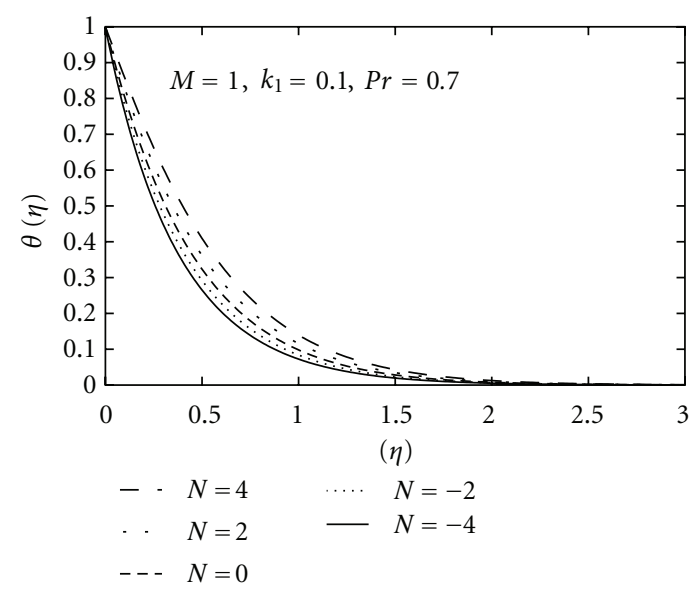

(c)

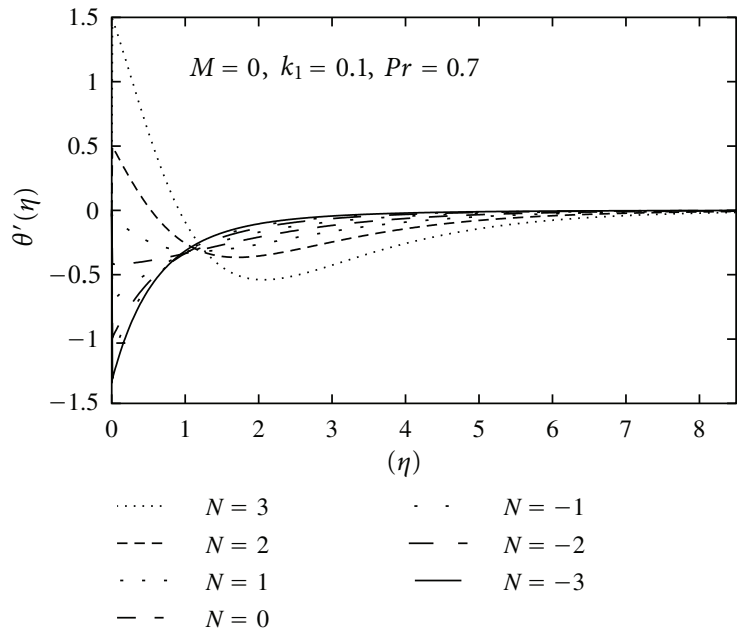

(b)

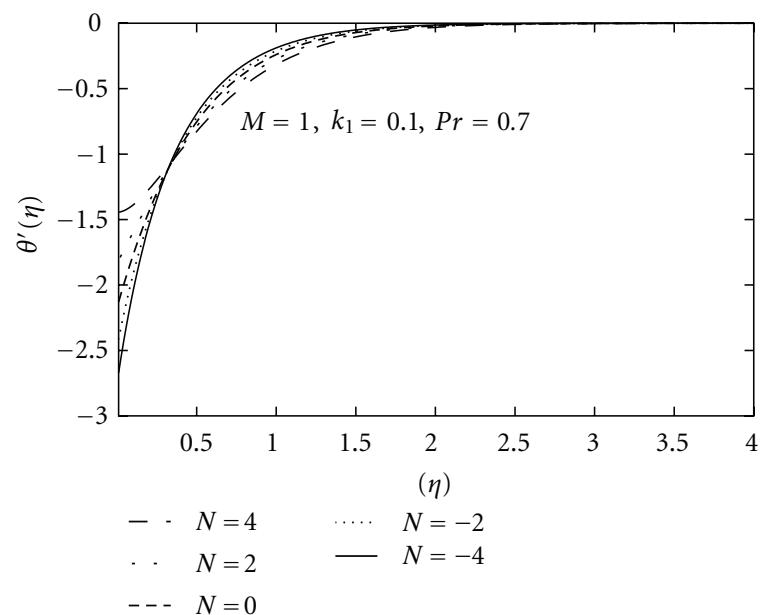

(d)

FIgURE 3: (a) Variation of temperature $\theta(\eta)$ with $\eta$ for several values of temperature exponent $N$ for flat plate with $M=0$. (b) Variation of temperature gradient $\theta^{\prime}(\eta)$ with $\eta$ for several values of temperature exponent, $N$ for flat plate with $M=0$. (c) Variation of temperature $\theta(\eta)$ with $\eta$ for several values of temperature exponent $N$ for stretching cylinder with $M=1$. (d) Variation of temperature gradient $\theta^{\prime}(\eta)$ with $\eta$ for several values of temperature exponent $N$ for stretching cylinder with $M=1$.

with the boundary conditions

$$
f(0)=0, \quad f^{\prime}(0)=1, \quad \theta(0)=1 .
$$

In order to integrate $(8)$ as initial value problems, one requires a value for $p(0)$, that is, $f^{\prime \prime}(0)$ and a value for $q(0)$ that is, $\theta^{\prime}(0)$ but no such values are given at the boundary. The suitable guess values for $f^{\prime \prime}(0)$ and $\theta^{\prime}(0)$ are chosen and then the integration is carried out. Comparing the calculated values for $f^{\prime}$ and $\theta$ at $\eta=10$ (say) with the given boundary conditions $f^{\prime}(10)=0$ and $\theta(10)=0$ and adjusting the estimated values, $f^{\prime \prime}(0)$ and $\theta^{\prime}(0)$, a better approximation for the solution is given.

Taking the series of values for $f^{\prime \prime}(0)$ and $\theta^{\prime}(0)$ and applying the fourth-order classical Runge-Kutta method with step size $h=0.01$, the above procedure is repeated until the results up to the desired degree of accuracy $\left(10^{-5}\right)$ are obtained.

\section{Results and Discussions}

In order to analyse the results, numerical computation has been carried out using the method described in the previous section for various values of the curvature parameter $(M)$, permeability parameter $\left(k_{1}\right)$, temperature exponent $(N)$ and Prandtl number (Pr). For illustrations of the results, numerical values are plotted in Figures 1(a) to 5(b).

For the verification of accuracy of the applied numerical scheme, a comparison of the present results corresponding to the heat transfer coefficient $\left[-\theta^{\prime}(0)\right]$ for $k_{1}=0$ (i.e., in case of nonporous medium) and $M=0$ (i.e., for stretching flat plate) with the available published results of Ishak and Nazar 


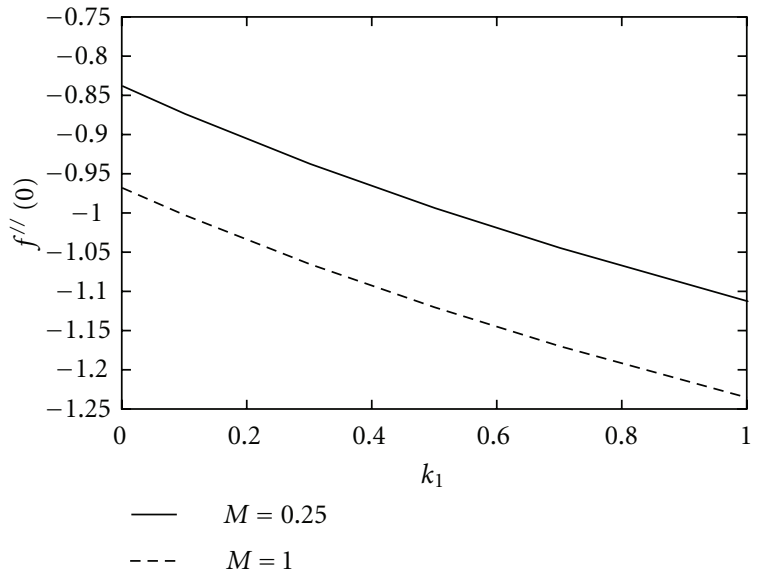

(a)

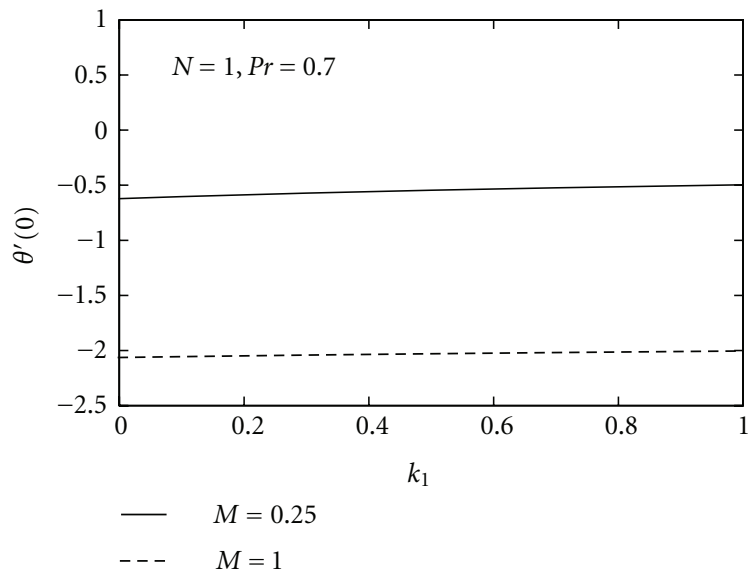

(b)

Figure 4: (a) Variation of skin friction coefficient $f^{\prime \prime}(0)$ with permeability parameter $k_{1}$ for two values of curvature parameter $M$. (b) Variation of heat transfer coefficient $\theta^{\prime}(0)$ with permeability parameter $k_{1}$ for two values of curvature parameter $M$.

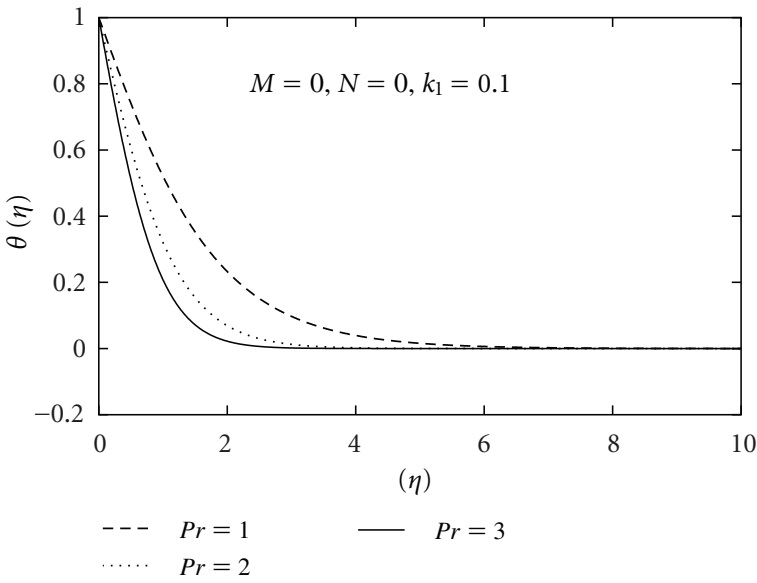

(a)

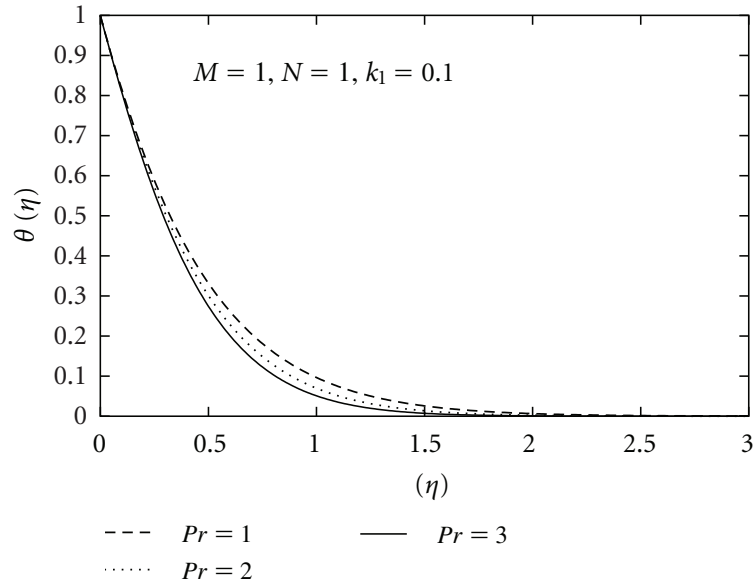

(b)

Figure 5: (a) Variation of temperature $\theta(\eta)$ with $\eta$ for several values of Prandtl number Pr for flat plate with $M=0$. (b) Variation of temperature $\theta(\eta)$ with $\eta$ for several values of Prandtl number Pr for stretching cylinder with $M=1$.

[17], Grubka and Bobba [18], Ali [19] is made and presented in Table 1. The results are found in excellent agreement.

Let us first concentrate on the effects of curvature parameter $M$ on velocity distribution in presence of porous medium. In Figure 1(a), horizontal velocity profiles are shown for different values of $M$. The horizontal velocity curves show that the rate of transport decreases with the increasing distance $(\eta)$ of the sheet. In all cases, the velocity vanishes at some large distance from the sheet (at $\eta=10$ ). The velocity increases with increasing values of $M$. The velocity gradient at the surface is larger for larger values of $M$, which produces larger skin friction coefficient.

Effects of curvature parameter on the temperature distribution are presented in Figure 1(b). Temperature is found to decrease with the increasing curvature parameter $M$. The thermal boundary layer thickness decreases as $M$ increases, which implies increasing in the wall temperature gradient and in turn increase the surface heat transfer rate. Hence, the Nusselt number increases as $M$ increases.

Now, the velocity profiles are presented for the variation of permeability parameter for flat plate and stretching cylinder, respectively. Figures $2(\mathrm{a})$ and $2(\mathrm{c})$ demonstrate the effects of permeability parameter $\left(k_{1}\right)$ for a stretching flat plate (i.e., for $M=0$ ) on velocity and temperature respectively. With the increasing $k_{1}$, the horizontal velocity is found to decrease (Figure 2(a)) but the temperature increases in this case. This feature prevails up to certain heights and then the process is slowed down. It is noted that $k_{1}$ has a substantial effect on the solutions. It is obvious that the presence of porous medium causes higher restriction to the fluid, which reduces the fluid velocity (Figure 2(a)) and enhances the temperature (Figure 2(b)). 
TABLE 1: Values of $N u_{x} \operatorname{Re}_{x}^{-1 / 2}=-\theta^{\prime}(0)$ for several values of temperature exponent $N$ for flat plate $(M=0)$ in nonporous medium $\left(k_{1}=0\right)$ and $\operatorname{Pr}=1$.

\begin{tabular}{lcccc}
\hline$N$ & $\begin{array}{c}\text { Ishak and } \\
\text { Nazar [17] }\end{array}$ & $\begin{array}{c}\text { Grubka } \\
\text { and Bobba } \\
{[18]}\end{array}$ & Ali [19] & $\begin{array}{c}\text { Present } \\
\text { study }\end{array}$ \\
\hline 0 & 0.5820 & 0.5820 & 0.5801 & 0.5821 \\
1 & 1.0000 & 1.0000 & 0.9961 & 1.0000 \\
2 & 1.3333 & 1.3333 & 1.3269 & 1.3332 \\
\hline
\end{tabular}

The porous medium presents resistance to the flow, thus, the flow becomes slower. Therefore, as the inverse Darcy number $\left(k_{1}^{-1}\right)$ increases, the resistance due to the porous medium increases and the surface velocity gradient increases. It is seen from the figure that the skin friction increases monotonically. $k_{1}=0$ corresponds to the case of nonporous medium. Figures $2(\mathrm{~b})$ and $2(\mathrm{~d})$ present the effects of permeability parameter $k_{1}$ on velocity and temperature for the stretching cylinder $(M>0)$. Here also velocity decreases with $k_{1}$ whereas the temperature increases with increasing $k_{1}$ (Figure 2(d)).

Figures 3(a) and 3(b) represent the temperature and temperature gradient profiles for variable temperature exponent $N$ for stretching flat plate $(M=0)$ in the presence of porous medium. It is seen that temperature increases with increasing values of $N$ and temperature overshoot is noted near the plate for $N>0$. The temperature gradient initially increases with $N$ but after a certain distance $\eta$ from the wall it decreases. Far away from the wall, such feature is smeared out. It is observed that, for higher positive values of $N(=$ $2,3)$, initially $\theta^{\prime}(\eta)$ is positive but finally it becomes negative. Temperature increases with $N$ but no temperature overshoot is noted for stretching cylinder $(M=1)$ (Figure 3(c)). For stretching cylinder, the temperature gradient is negative for all values of $N$ (Figure 3(d)).

Figures 4(a) and 4(b) present the behaviour of skin friction and heat transfer coefficients with the permeability parameter $k_{1}$ of the porous medium for two values of curvature parameter. Magnitude of the skin friction coefficient increases with increasing permeability parameter $k_{1}$ and also with the curvature parameter $M$. This feature also supports the earlier findings in Figures 1(a), 2(a) and 2(b). From the figure, it is very clear that shear stress at the wall is negative here. Physically, negative sign of $f^{\prime \prime}(0)$ implies that surface exerts a dragging force on the fluid and positive sign implies the opposite. This is consistent with the present case as a stretching cylinder which induces the flow is considered here. From Figure 4(b) it is very clear that the magnitude of heat transfer coefficient decreases with permeability parameter $k_{1}$ as well as with the curvature parameter $M$. This is consistent with the findings in Figures 1(b), 2(c) and 2(d).

It is noted that temperature decreases with increasing Pr. An increase in Prandtl number reduces the thermal boundary layer thickness. It is also observed that the effects of Pr is much more prominent for flat plate (Figure 5(a)) compared to stretching cylinder (Figure 5(b)). Prandtl number signifies the ratio of momentum diffusivity to thermal diffusivity.
Fluids with lower Prandtl number will possess higher thermal conductivities (and thicker thermal boundary layer structures) so that heat can diffuse from the wall, faster than for higher Pr fluids (thinner boundary layers). Hence, Prandtl number can be used to increase the rate of cooling in conducting flows.

\section{Conclusions}

The present study gives the numerical solutions for steady boundary layer flow and heat transfer along a stretching cylinder embedded in porous medium. The rate of transport is considerably reduced with increasing values of curvature parameter $M$. The results pertaining to the present study indicate that due to increasing permeability parameter, velocity decreases. The surface shear stress and the heat transfer rate at the surface increase as the curvature parameter increases.

It is hoped that, the physics of flow over the stretching cylinder can be utilized as the basis for many engineering and scientific applications with the help of the present model. The results pertaining to the present study may be useful for the different model investigations. The findings of the present problem are also of great interest in different areas of science and technology where the surface layers are being stretched.

\section{References}

[1] L. J. Crane, "Flow past a stretching plate," Zeitschrift für Angewandte Mathematik und Physik, vol. 21, no. 4, pp. 645-647, 1970.

[2] P. S. Gupta and A. S. Gupta, "Heat and mass transfer on a stretching sheet with suction or blowing," The Canadian Journal of Chemical Engineering, vol. 55, no. 6, pp. 744-746, 1977.

[3] B. K. Dutta, P. Roy, and A. S. Gupta, "Temperature field in flow over a stretching sheet with uniform heat flux," International Communications in Heat and Mass Transfer, vol. 12, no. 1, pp. 89-94, 1985.

[4] C. K. Chen and M. I. Char, "Heat transfer of a continuous, stretching surface with suction or blowing," Journal of Mathematical Analysis and Applications, vol. 135, no. 2, pp. 568-580, 1988.

[5] H. Xu and S. J. Liao, "Series solutions of unsteady magnetohydrodynamic flows of non-Newtonian fluids caused by an impulsively stretching plate," Journal of Non-Newtonian Fluid Mechanics, vol. 129, no. 1, pp. 46-55, 2005.

[6] R. Cortell, "Flow and heat transfer of a fluid through a porous medium over a stretching surface with internal heat generation/absorption and suction/blowing," Fluid Dynamics Research, vol. 37, no. 4, pp. 231-245, 2005.

[7] R. Cortell, "Effects of viscous dissipation and work done by deformation on the MHD flow and heat transfer of a viscoelastic fluid over a stretching sheet," Physics Letters A, vol. 357, no. 4-5, pp. 298-305, 2006.

[8] T. Hayat, Z. Abbas, and M. Sajid, "Series solution for the upper-convected Maxwell fluid over a porous stretching plate," Physics Letters A, vol. 358, no. 5-6, pp. 396-403, 2006.

[9] T. Hayat and M. Sajid, "Analytic solution for axisymmetric flow and heat transfer of a second grade fluid past a stretching 
sheet," International Journal of Heat and Mass Transfer, vol. 50, no. 1-2, pp. 75-84, 2007.

[10] P. Datta, D. Anilkumar, S. Roy, and N. C. Mahanti, "Effect of non-uniform slot injection (suction) on a forced flow over a slender cylinder," International Journal of Heat and Mass Transfer, vol. 49, no. 13-14, pp. 2366-2371, 2006.

[11] E. M. E. Elbarbary and N. S. Elgazery, "Flow and heat transfer of a micropolar fluid in an axisymmetric stagnation flow on a cylinder with variable properties and suction (numerical study)," Acta Mechanica, vol. 176, no. 3-4, pp. 213-229, 2005.

[12] M. Kumari and G. Nath, "Mixed convection boundary layer flow over a thin vertical cylinder with localized injection/ suction and cooling/heating," International Journal of Heat and Mass Transfer, vol. 47, no. 5, pp. 969-976, 2004.

[13] C. Y. Wang, "Fluid flow due to a stretching cylinder," Physics of Fluids, vol. 31, pp. 466-468, 1988.

[14] A. Ishak, R. Nazar, and I. Pop, "Uniform suction/blowing effect on flow and heat transfer due to a stretching cylinder," Applied Mathematical Modelling, vol. 32, no. 10, pp. 20592066, 2008.

[15] H. T. Lin and Y. P. Shih, "Laminar boundary layer heat transfer along static and moving cylinders," Journal of the Chinese Institute of Engineers, vol. 3, no. 1, pp. 73-79, 1980.

[16] H. T. Lin and Y. P. Shih, "Buoyancy effects on the laminar boundary layer heat transfer along vertically moving cylinders," Journal of the Chinese Institute of Engineers, vol. 4, no. 1, pp. 47-51, 1981.

[17] A. Ishak and R. Nazar, "Laminar boundary layer flow along a stretching cylinder," European Journal of Scientific Research, vol. 36, no. 1, pp. 22-29, 2009.

[18] L. J. Grubka and K. M. Bobba, "Heat transfer characteristics of a continuous stretching surface with variable temperature," Journal of Heat Transfer, vol. 107, no. 1, pp. 248-250, 1985.

[19] M. E. Ali, "Heat transfer characteristics of a continuous stretching surface," Heat and Mass Transfer, vol. 29, no. 4, pp. 227-234, 1994.

[20] A. Ishak, R. Nazar, and I. Pop, "Steady and unsteady boundary layers due to a stretching vertical sheet in a porous medium using Darcy-Brinkman equation model," International Journal of Applied Mechanics and Engineering, vol. 11, no. 3, pp. 623637, 2006. 

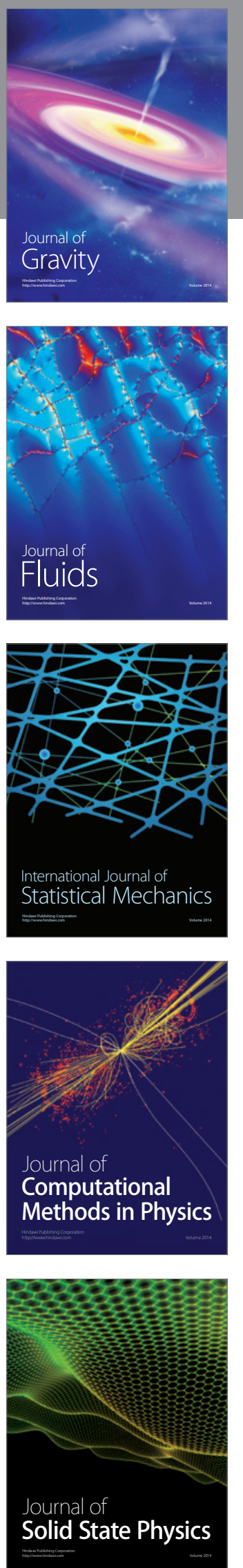
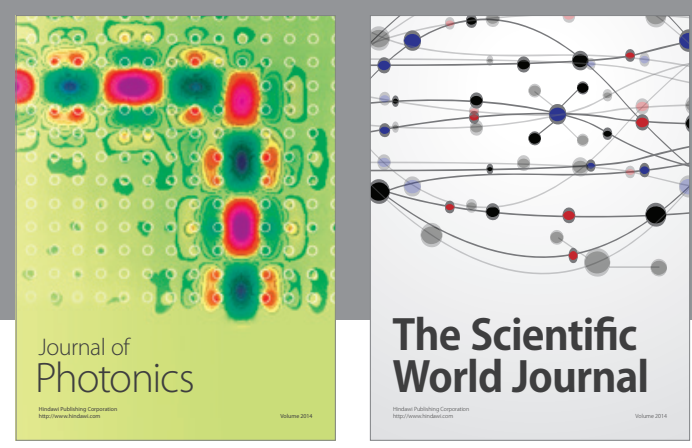

The Scientific World Journal



Submit your manuscripts at http://www.hindawi.com
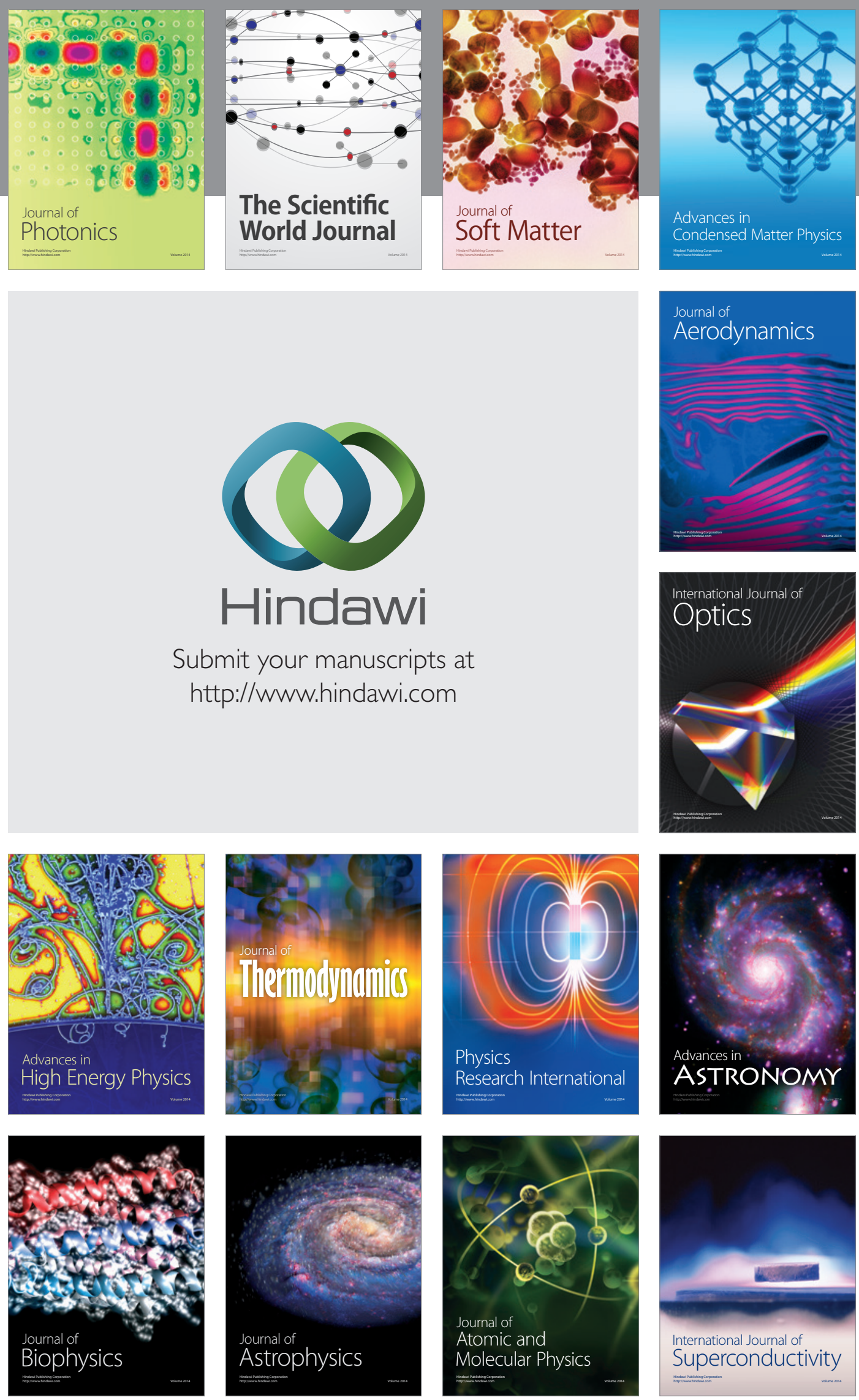
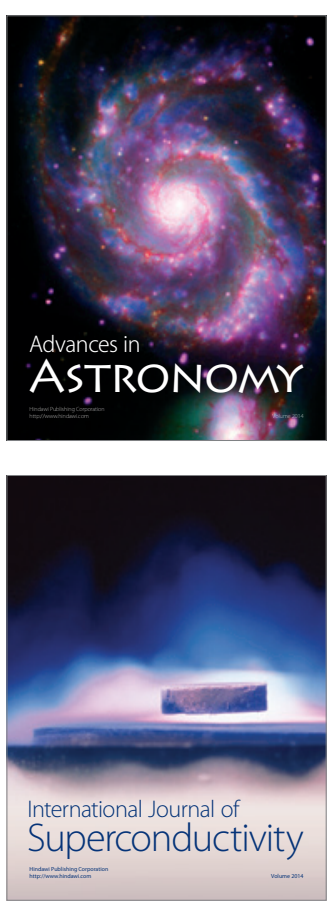